\title{
Development of a Bulldozer Operation Simulator
}

\author{
Shigeo Mimura, Yutaka Ishimatsu, Tadashi Yoshida \\ Public Works Research Institute,Ministry of Construction,1 Asahi,Tsukuba-shi,Ibaraki,
} 305 JAPAN

Bulldozers and similar general purpose earthmoving equipment frequently handle soil, work which must be done by operators with considerable experience. A study of an artificial intelligence technology based control system that can permit even inexperienced bulldozer operators to perform high quality earthwork has been carried out. This paper provides a summary of that study along with a report on a bulldozer operation simulator developed to provide a way to obtain operating data needed to recreate the working environment and define the operating objectives to be applied to the creation of a time-series model of bulldozer operation.

The simulator simplifies the bulldozer into a two-dimensional model and displays the position and attitude of the bulldozer in real time on a CRT screen in response to blade motion input. The study included the incorporation of the dynamics of the machine - ground system: the generation of drive force by the crawler and the cutting load applied to the blade for example.

\section{INTRODUCTION}

A bulldozer operator has to correctly assess changes in the inclination of the body of the machine, the slippage of the track assembly, and other operating conditions, and based on these assessments, make suitable judgements to operate the machine. But soil, which is both the material the operator is dealing with and the surface on which the bulldozer is moving, changes in complex ways as the work progresses, which means that skilful operation of a bulldozer in response to work conditions depends to a great degree on the operator's skill, or in other words, the degree of experience he has obtained.

This is one reason why it is difficult for inexperienced bulldozer operators to perform high quality work.

One idea proposed as a way to create an "Easy Operation System" for bulldozers in order to resolve this problem is the creation of a model of part of the observation of conditions, judgements, and operation tasks performed by bulldozer operators, and the incorporation of this model in a bulldozer control system.

Because it is assumed that the state of the supporting ground which varies the inclination of a bulldozer has a big influence on the hysteresis of the operation, during the modelling process, the operator's operating model is described based on interaction of the operation time history up 
to that point. So the study focussed on the development of a simulator that could repetitively collect data under highly reproducible conditions in order to gather the operation time history data needed to construct the operator's operation model. As the basic step in that process, an operation simulator based on a behavior calculation model of a simple machine-ground system in which the blade operating lever is an outside input device was developed.

\section{STUDY OF THE CHARACTERISTICS OF BULLDOZER OPERATION}

\subsection{Characteristics of the Man-Machine System}

When the operator's operating process was considered, it was concluded that it would be necessary to consider the modelling in terms of the hierarchical structure shown in Figure 1.

(1) Work Planning

The bulldozer operation plan is part of the overall quantity of work of an execution, and this plan is changed as necessary according to the quality of the soil to be handled. There are cases where it is impossible to perform the work according to the original work plan, so in order to deal with such situations, it is essential that the operator is constantly aware of changes in external conditions such as the topography, soil quality, etc. It is extremely difficult to do this with present day measuring technology, so realistically it must rely on the operator's observations of the situation.

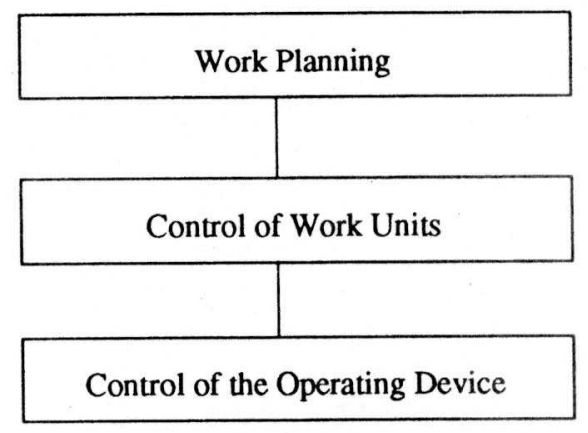

Figure 1

Structure of Bulldozer Work

\section{(2) Control of Work Units}

The part of bulldozer work that is most dependent upon the skills of an experienced operator, it refers to the movement and control of the blade during the performance of cutting, crowding, embanking, levelling, and other operations. Examples include the blade motion required to deal with the changed inclination of the bulldozer body as its tracks enter a part already cut by the blade, or the movement of the blade performed to deal with a change in the attitude of the bulldozer body during levelling work. But because this process includes remaining aware of work conditions such as changes in cutting resistance or the resulting crawler slippage and, as necessary, varying the amount of blade motion to compensate for these changes, there are limits of various kinds to linear control of this process.

(3) Control of the Operating Device

This can be referred to as servo control of the hydraulic cylinders that control blade position.

Based on the above study, an operator operation time history model was prepared by applying a neural network able to create a model based on case study data concerning input and output for one category of "work unit control," namely, "the process up to the 
point where the blade has begun cutting, its tracks have entered the cutting area, and constant cutting status is achieved."

\subsection{Characteristics of the Machine - Ground System}

A dynamic study focussed on the locomotion properties of a tracked vehicle and bulldozer operating characteristics was made of the ground material, track shape and other aspects of the ground - machine interaction in order to effectively utilize the ground bearing force and the drive power. ${ }^{1)}$

The tensile force transmitted to the tracks is a product of the rpm - torque performance of the drive system of the bulldozer. The parallel movement of the tracks over the ground surface produced by this tensile force generates shear resistance on the ground underneath the tracks to drive the bulldozer body under stress distribution conditions caused by the body load. The traction force acts on the body movement produced by the drive force. The ground resistance force is assumed to be compaction resistance and friction resistance. The compaction resistance is that produced as the body moves forward compaction the soil, and the friction resistance is caused by friction created by contact of the natural ground with the clay soil adhering to the under carriage of the tracks as the body moves forward. And as a result of the tilting of the bulldozer as it moves, the locomoting direction component of the bulldozer body weight acts as locomotion resistance.

Then when the blade is used to cut the soil, the blade is acted upon by the ground's resistance force generated by the cutting, but because the mechanism of the ground failure in front of the blade caused by the cutting is not fully understood and the soil quality is scattered according to the location and depth, it is difficult to decisively determine the cutting resistance from the blade shape or ground parameters. And when the blade is inserted into the ground, intrusion resistance is produced.

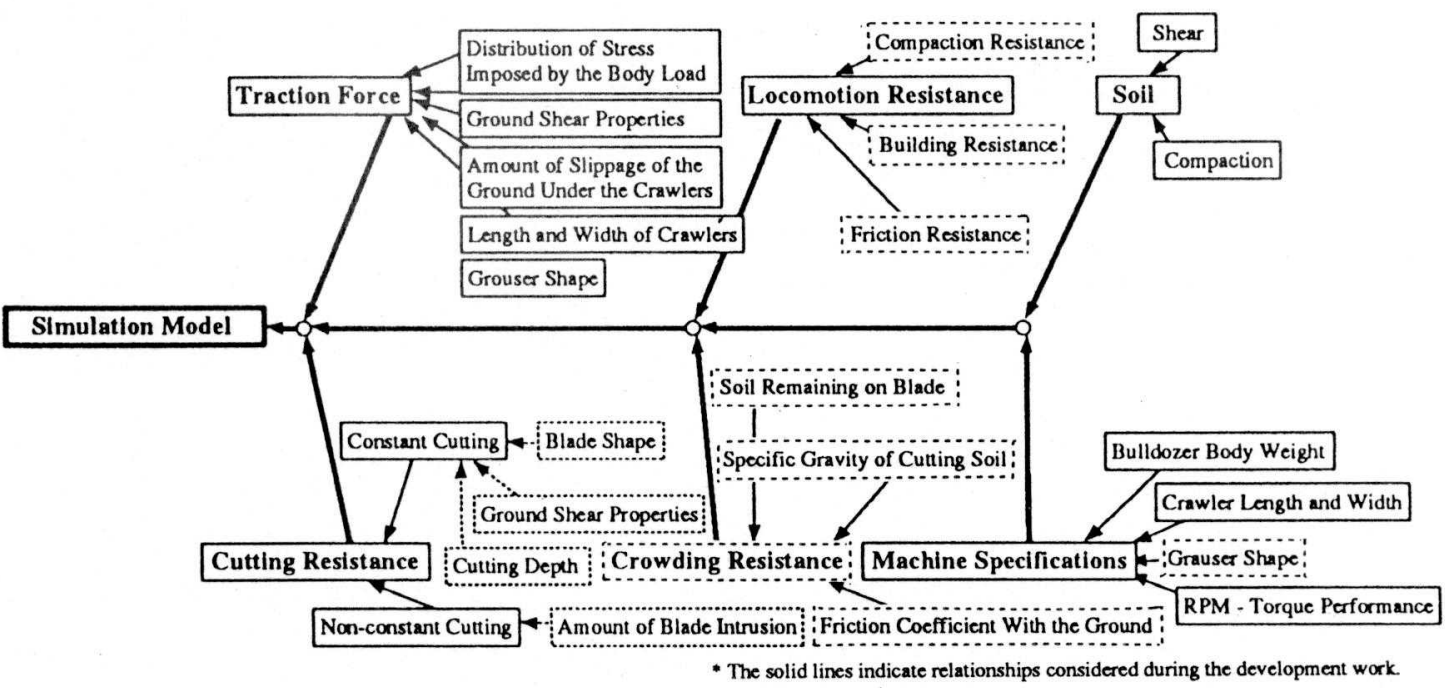

Figure 2 Bulldozer Characteristic Factor Diagram 
These are influenced by the body weight, location of its center of gravity, track width and length, grouser shape, the shape of the blade, other bulldozer specifications, as well as by the shape of the ground and the soil quality.

Figure 2 presents the results of a study and organization of factors which must be considered in the bulldozer model, including these other resistance forces.

\section{BULLDOZER OPERATION SIMULATOR}

The simulation model study was performed by finding the resistance caused by the cutting work under optional soil and ground shape conditions, and the traction force generated against this resistance force by the bulldozer, then representing the bulldozer behavior during its operation by performing a series of calculations of the body inclination and the amount of movement from the static balance of these forces.

\subsection{Setting the Forces Acting in the Bulldozer's Locomotive Direction}

The forces acting in the bulldozer's locomotive direction were set as shown in Figure 3 for the purpose of modelling the machine - ground system.

Where:

$\mathrm{T}$ : Traction force

$\mathrm{T}_{\mathrm{c}}$ : Cutting resistance

$\mathrm{T}:$ Locomotion resistance

$\mathrm{T}_{\mathrm{i}}$ : locomotion load produced by the body inclination

W: Body load

$\phi:$ Angle of inclination of the body

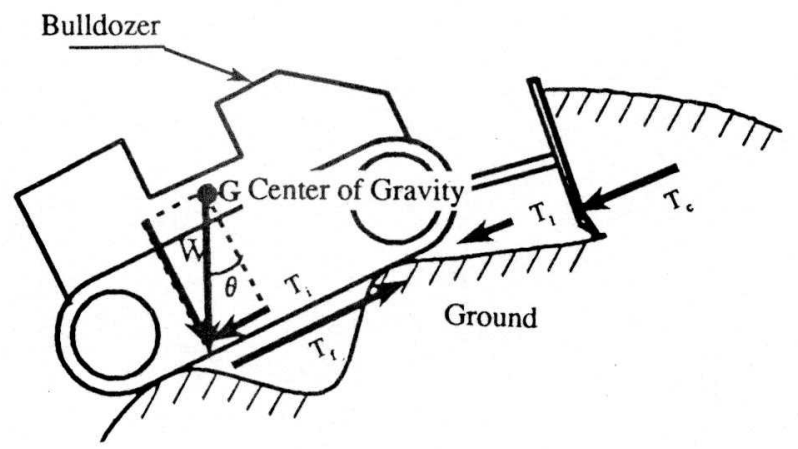

Figure 3 Forces Acting on a Bulldozer (Locomotive Direction)

\section{(1) Traction Force}

When a bulldozer locomotes on ground with depressions and projections on its surface, part of the tracks are moving in contact with the ground. So when the length of the track that is touching the ground was assumed to be $l_{c}$, the traction force $T_{t}$ which the bulldozer can produce was calculated as shown in the following formula.

However:

$$
\mathrm{T}_{\mathrm{t}}=\eta \cdot\left(2 \cdot \mathrm{c} \cdot \mathrm{d}_{\mathrm{c}} \cdot \mathrm{l}_{\mathrm{c}}+\mathrm{W} \tan \phi\right)
$$

$\eta$ : Compensation coefficient $\quad \mathrm{c}$ : Cohesion of the ground

W: Body weight

$\mathrm{d}_{\mathrm{c}}$ : Track width

$\phi:$ Internal friction angle of the ground 
The compensation coefficient $\eta$ accounted for the fact that the ground surface soil quality is actually not homogenous, and for the distribution shape of the contact pressure of the ground. The above formula finds the shearing resistance for the entire contact surface of the tracks based on Mohr-Coulomb's standard formula.

\section{(2) Cutting Resistance and Locomotion Resistance}

The cutting resistance force $T_{c}$ is assumed to cause shear stress in the sliding surface produced in the ground in front of the blade as the blade moves forward. However, the sliding surface was simplified by treating it as a straight line for the calculation. As seen in Figure 4, it was assumed that a sliding surface was produced at an angle of $\alpha$ to the locomotive direction in the ground in front of the blade, and that the sliding surface length $\mathrm{L}_{\mathrm{s}}$ changed according to the angle of inclination $\theta$ of the body and the shape of the ground. Figure 5 shows the calculation flow chart. In the flow chart, $y_{o}$ represents the height of the bottom edge of the blade, $\Delta X$ represents the calculation interval of the topographical data, and $h_{i}$ represents topographical data corresponding to No. $\mathrm{i}$ in front of bottom edge of the blade.

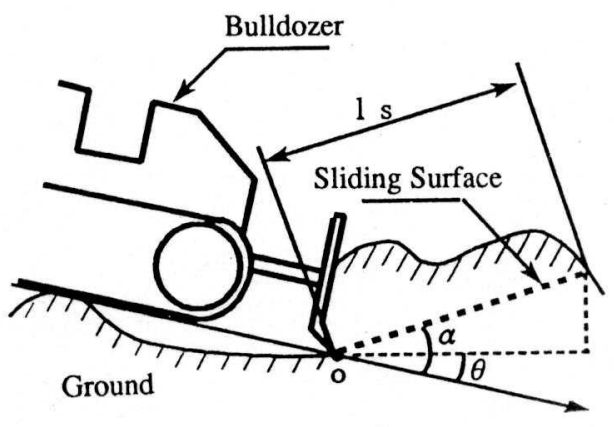

Figure 4 Principle of the Sliding Surface Based on Shear

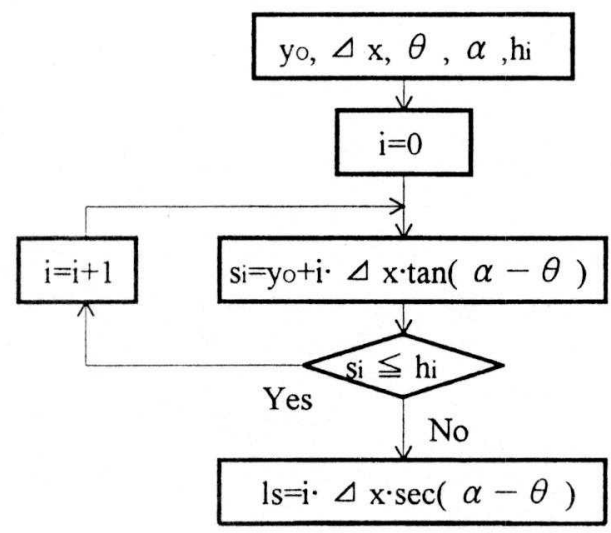

Figure 5 Sliding Surface Length Calculation Flow Chart

The cutting resistance force was calculated with $d_{b}$ as the blade width and $l_{s} \cdot d_{b}$ as the size of the shear plane generated in the ground in front of the blade. As for the other resistance forces, the locomoting load $\mathrm{T}_{\mathrm{i}}$ caused by the inclination 'of the body was combined with the compaction resistance produced by the bulldozer moving as its crawler left depressions in the ground, the crowding resistance caused by cut soil remaining on the front surface of the blade, and other resistance forces, and these combined resistance forces were accounted for by treating the locomotion resistance $T_{1}$ as a constant.

The slippage between the crawler and the ground surface was accounted for by assuming that in a case where the resistance forces against the locomotion of the bulldozer and the cutting are either identical to or exceed the maximum tractive force which the ground can provide, the slippage rate is " 1 ", or in other words, the crawler is completely slipping against the ground, and assuming that the tractive ratio is $H=\left(T_{c}+T_{1}+T_{i}\right) / T_{t}$, the slippage rate $i$ is calculated using the following formula.

$$
\mathrm{i}=\mathrm{H}^{\mathrm{k}}
$$




\subsection{Body Attitude Numerical Calculation Method}

The bulldozer attitude was calculated with the tracks assumed to be rigid tracks and the ground bearing the body treated as a rigid body.

The attitude of the bulldozer is determined based on two points of contact between the ground and the track surface, one behind and one in front of the center, which is assumed to be the point that the body load on the center of gravity acts on the track surface.

The basic approach to finding the coordinates of these two points is to sequentially compare the inclinations of lines linking adjoining points of the topographical data in either the forward or rearward direction from the location of the center of gravity, and while eliminating the point at the lower location from the process, repeating the process to the front and back ends of the track. In this way, two contact points on the track, one in front of and one behind the center of gravity, are determined. Figure 6 shows a sample calculation used to find the forward contact point $f$ from the location of the center of gravity of the body of the bulldozer.
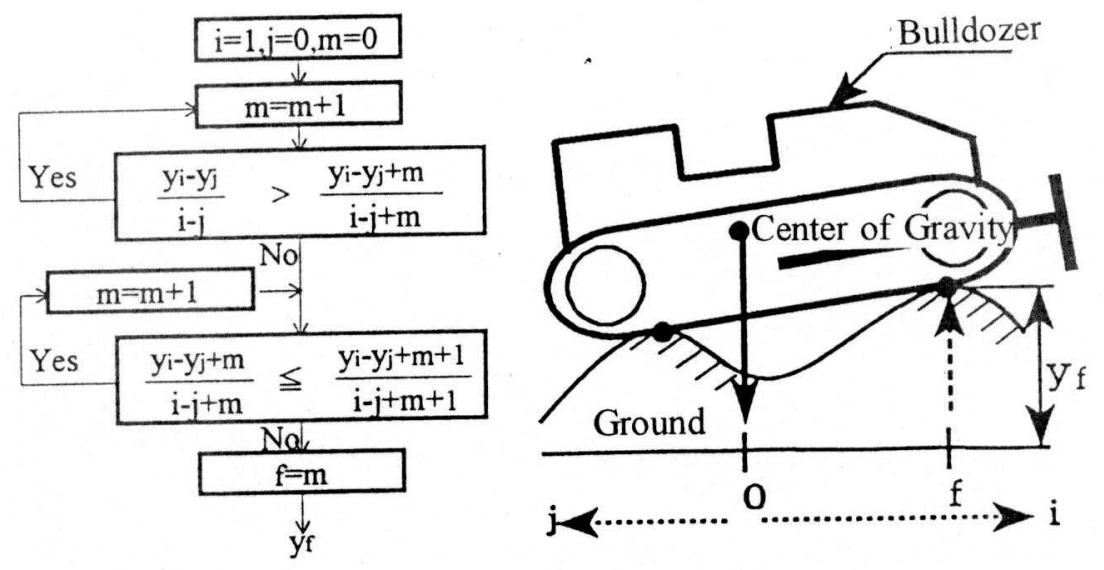

Figure 6 Ground Contact Point Calculation Method

\subsection{System Structure}

The simulator equipment includes a personal computer and a joy stick that serves as the bulldozer operation input device. The program includes [1] body attitude calculation routine, [2] traction force calculation routine, [3] cutting resistance force calculation routine, [4] body movement speed calculation routine, and [5] screen display routine needed to display the bulldozer and topography. Figure 7 and Figure 8 show the overall program structure and a sample simulator screen respectively.

\subsection{Operation Example}

To study its conformity with the operation of an actual bulldozer, the cutting objectives were determined by establishing test conditions in accordance with the results of measurements of actual bulldozer work; the cutting depth was set at $0.2 \mathrm{~m}$, and the angle of insertion of the blade was assumed to equal $1 / 10$ of the distance moved by the bulldozer. The operator manipulated 
the joy stick, which served as the blade operation lever so that the blade would follow the cutting target values shown on the display screen. The experiment was performed after the operator had practiced operating the simulator until he was able to operate it skillfully. Figure 9 shows the finished work achieved by the simulator. Figure 10 presents changes in the inclination of the body during the work.

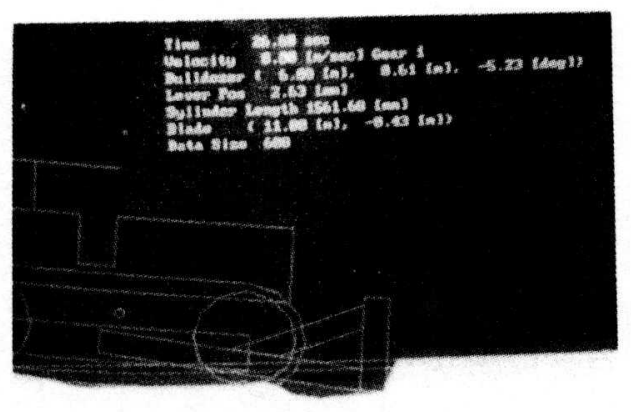

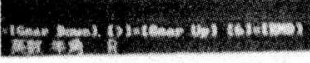

Figure 7 Simulation Screen

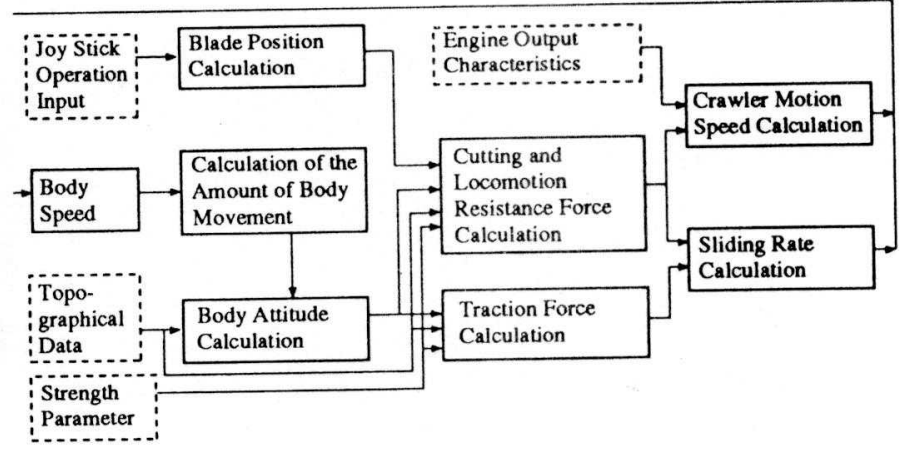

Figure 8 Program Structure

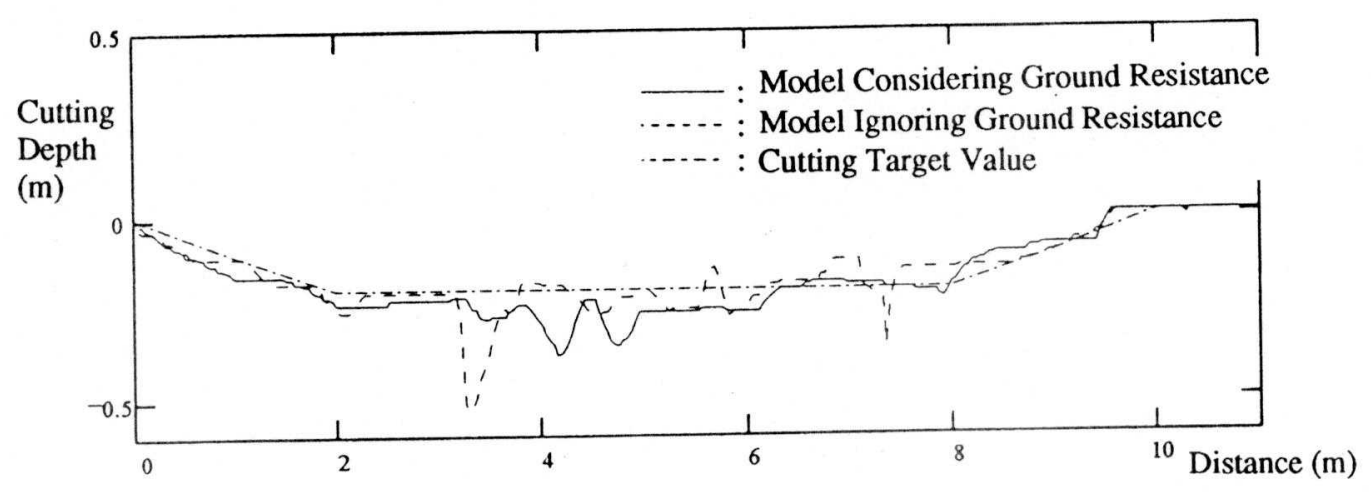

Figure 9. Completed Ground After the Work

\section{SUMMARY}

The following results were obtained from this research project.

(1) Figure 9 shows that in the case of the model which did not consider the ground resistance, conspicuous ground irregularities were formed near the points where the bulldozer body entered the cutting area, but that the newly developed simulator resolved the problem. This happened because the simulation accounted for the ground resistance against the intrusion of the blade. 
(2) The irregularities seen in the ground in the cutting area in Figure 9, which were the result of the failure to account for the effects of the crowding produced by the tracks on the ground where the bulldozer locomoted, are very conspicuous when compared with the actual work. It will be possible to apply future terramechanic theory to permit later simulators to account for the factors not accounted for by the simulator developed by this research project.

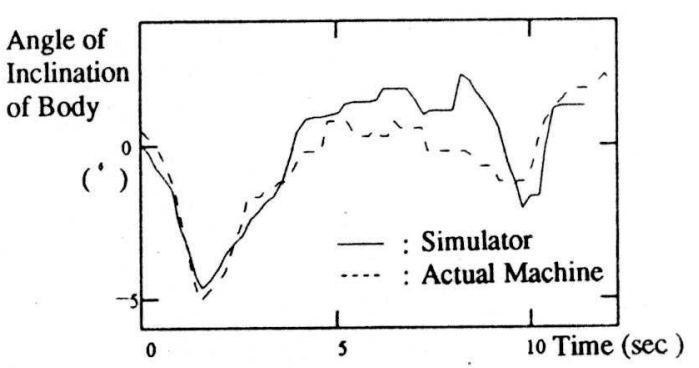

Figure 10 Operating Example (Body Inclination Changes)

(3) Figure 10, which shows changes in the inclination of the body while the bulldozer cut the ground with its blade as it locomoted over the ground, confirms that this simulator follows the body inclination caused by changes in the ground shape, just as if it were a real machine.

(4) This simulator found the amount of slippage between the ground and the tracks from the static balance relationship of the traction force and the resistance force of the cutting etc. If it is eventually possible to account for the acceleration properties of the bulldozer by entering these forces, it will also be possible to develop a simulator that permits the behavior during acceleration and braking to be handled more realistically.

\section{CONCLUSION}

Through past research projects, the feasibility of applying artificial intelligence to develop a bulldozer operating support method has been studied. The simulator developed through this last project was based on the hypothesis that a neural network model of an operator operating a bulldozer would be prepared from operating data. This is a fundamental stage in the study of an easy operation system for bulldozers; the ultimate objective of this research program.

In conclusion, we would like to express our deep gratitude to Professor Yuta and Mr. Kimoto of Tsukuba University and to Mr. Ichikawa of Shimizu Construction for their generous guidance and assistance with this simulator development project.

\section{Sources}

1) Muro Tatsuro: Telemechanics - Locomotion Dynamics, Gihodo Shuppan, 1993.

2) Japan Construction Mechanization Association: Papers Presented at the Automation Committee Conference "New Approaches to Construction Robots," 1992.

3) JIP DEC: Uses of AI Technology, 1988. 